\title{
Vibration of an adhered microbeam under a periodically shaking electrical force
}

\section{YIN ZHANG and YA-PU ZHAO*}

State Key Laboratory of Nonlinear Mechanics (LNM), Institute of Mechanics, Chinese Academy of Sciences, Beijing 100080, People's Republic of China

Received in final form 14 May 2005

\begin{abstract}
The vibration analysis of an adhered S-shaped microbeam under alternating sinusoidal voltage is presented. The shaking force is the electrical force due to the sinusoidal voltage. During vibration, both the microbeam deflection and the adhesion length keep changing. The microbeam deflection and adhesion length are numerically determined by the iteration method. As the adhesion length keeps changing, the domain of the equation of motion for the microbeam (unadhered part) changes correspondingly, which results in changes of the structure natural frequencies. For this reason, the system can never reach a steady state. The transient behaviors of the microbeam under different shaking frequencies are compared. We deliberately choose the initial conditions to compare our dynamic results with the existing static theory. The paper also analyzes the changing behavior of adhesion length during vibration and an asymmetric pattern of adhesion length change is revealed, which may be used to guide the dynamic de-adhering process. The abnormal behavior of the adhered microbeam vibrating at almost the same frequency under two quite different shaking frequencies is also shown. The Galerkin method is used to discretize the equation of motion and its convergence study is also presented. The model is only applicable in the case that the peel number is equal to 1 . Some other model limitations are also discussed.
\end{abstract}

Keywords: Microbeam; vibration; adhesion; peel number; electrical force; quasistatic.

\section{INTRODUCTION}

The voltage between a microelectromechanical systems (MEMS) structure and the substrate is an important issue for a capacitor-like MEMS structure [1-9]. Due to the attractive force generated by the voltage, a free-standing structure may experience pull-in instability which finally may lead to structure adhering to the substrate [5-8]. For the adhered structure, the static voltage also affects both the

*To whom correspondence should be addressed. Tel.: (86-10) 6265-8008. Fax: (86-10) 6256-1284. E-mail: yzhao@lnm.imech.ac.cn 
microbeam deflection as well as adhesion length [9]. Mastrangelo and Hsu [10, 11] used the energy method which determines the structure equilibrium/equilibria by finding the energy functional stationary point(s) for the static studies of the different structures adhesion problems under no electrical force influence. So far, most studies have focused on the static study of the adhesion problem [4, 9-14]. This paper extends Mastrangelo and Hsu's energy method to the dynamic case of the adhered microbeam vibration under an electrical shaking force by incorporating the kinetic energy and the work due to the electrical force into the energy functional. The microbeam is divided into two parts: the adhered part and the unadhered part. The adhered part is assumed flat and immovable and, therefore, it does not contribute to either elastic potential energy or kinetic energy of the beam. For the unadhered part, its deflection is determined by the work of electrical force, the elastic potential energy and kinetic energy. The derivative of energy functional, which includes the kinetic, and potential energies, work of electrical force and adhesion, is used to determine the microbeam adhesion length. In each time step, the derivative is taken to determine whether the microbeam peels-off or adheres more. Once the stationary point(s) is found, and the adhesion length is determined, the microbeam deflection is re-computed. On the other hand, the microbeam deflection and adhesion length must be assumed at the beginning to compute the energy functional. The iteration is used to determine the microbeam deflection and adhesion length in each time step. The goal is to provide an approach to study the vibration of an adhered structure. Although the excitation source is the electrical force in this study, it can be extended to study the vibration due to the other sources such as mechanical, inertial, or acoustic excitation.

\section{PHYSICAL MODEL AND PROBLEM FORMULATION}

In Fig. 1a, a microbeam with length $L$ is separated from the substrate by a gap distance $g$. The substrate is coated with a thin dielectric layer of thickness $d_{\mathrm{e}}$ with permittivity $\epsilon$. Figure $1 \mathrm{~b}$ and $1 \mathrm{c}$ shows the arch-shaped and S-shaped adhered microbeams, respectively. For the arch-shaped microbeam, the contact area is extremely small; therefore, the measurement largely depends on the contact local properties and the statistical error is relatively much higher [4]. de Boer et al. [15] argue that a better way of studying meaningful surface interaction energy is to investigate the S-shaped case only. Thus, in this paper, only the S-shaped adhered microbeam is studied. $S$ is the length of the unadhered part and $L-S$ is the adhesion length of the $\mathrm{S}$-shaped microbeam.

In Fig. 1c, the kinetic energy of the unadhered beam part is given as

$$
T=\frac{1}{2} \int_{0}^{S} \rho w_{t}^{2} \mathrm{~d} x,
$$

where $\rho$ is the microbeam mass per unit length. $w$ is the microbeam deflection and $w=w(t, x, S) . w_{t}=\partial w / \partial t$ and $t$ is time. It is assumed that there is no motion in 


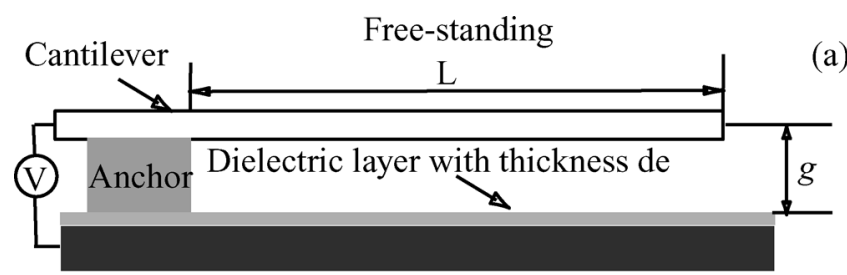

(a)

Substrate

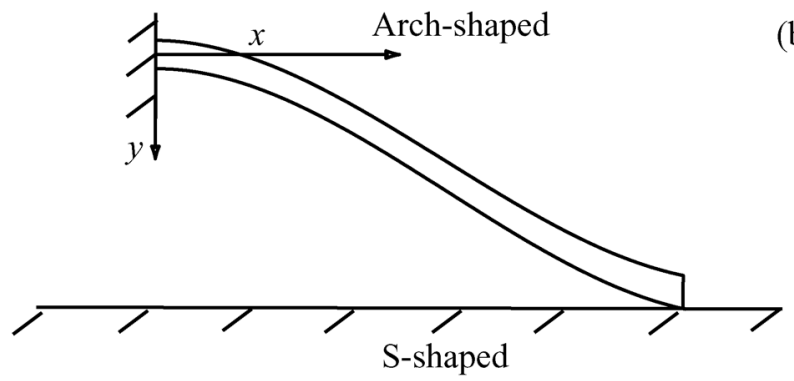

(b)

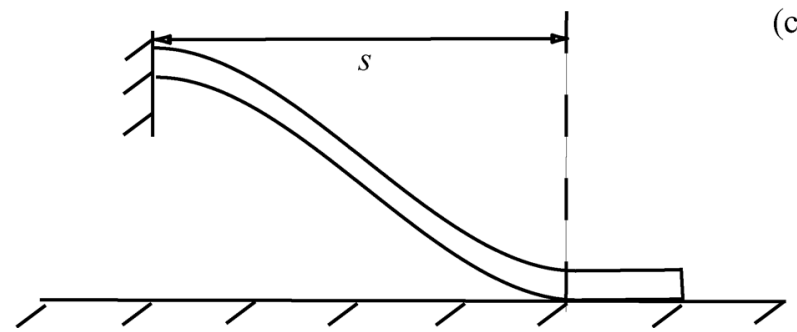

(c)

Figure 1. (a) The schematic diagram of microbeam-substrate (with dielectric layer) system and structure dimensions. (b) and (c) The arch-shaped and S-shaped stiction behaviors, respectively. The coordinate system is shown in (b).

the adhered part. $U_{\mathrm{B}}$, the microbeam elastic potential energy of bending, is given as

$$
U_{\mathrm{B}}=\frac{E I}{2} \int_{0}^{S}\left(\frac{\partial^{2} w}{\partial x^{2}}\right)^{2} \mathrm{~d} x
$$

where $E I$ is microbeam bending stiffness, $E$ is Young's modulus and $I=b h^{3} / 12$ ( $b$ and $h$ are the beam width and thickness, respectively) for rectangular cross section beam. For the adhered part, the microbeam stays flat and there is no deformation, so its elastic potential energy is zero. $U_{S 1}$, the stretching energy part due to the large deformation, is [16]

$$
U_{S 1}=\frac{A E}{2 S}\left\{\frac{1}{2} \int_{0}^{S}\left(\frac{\partial w}{\partial x}\right)^{2} \mathrm{~d} x\right\}^{2},
$$


where $A$ is the cross-sectional area and $A=b h . U_{S 2}$, the microbeam stretching energy part due to the axial load $P$, is as follows

$$
U_{S 2}=\frac{P}{2} \int_{0}^{S}\left(\frac{\partial w}{\partial x}\right)^{2} \mathrm{~d} x
$$

$W_{\mathrm{S}}$, the detachment work due to adhesion is [17]

$$
W_{\mathrm{S}}=b(L-S) f_{\theta} W_{\mathrm{a}},
$$

where $f_{\theta}$ is a dimensionless roughness function and $W_{\mathrm{a}}$ is the Dupré adhesion or work of adhesion per unit area, expressed as [18]

$$
W_{\mathrm{a}}=\gamma_{1}+\gamma_{2}-\gamma_{12},
$$

where $\gamma_{1}$ and $\gamma_{2}$ are the two surface tensions of the beam and substrate before adhesion, respectively. $\gamma_{12}$ is the interfacial tension after adhesion and $\gamma_{1}+\gamma_{2}-$ $\gamma_{12}>0$, which physically means that when the two surfaces unite to form an interface, the net free energy reduces.

$W_{\mathrm{E}}$, the work done by $f_{\mathrm{e}}$, the electrical force per unit length, is

$$
W_{\mathrm{E}}=\int_{0}^{S} \int_{0}^{w} f_{\mathrm{e}} \mathrm{d} w \mathrm{~d} x
$$

$f_{\mathrm{e}}$ has the following expression for the substrate coated with a layer of dielectric material $[1,2]$.

$$
f_{\mathrm{e}}=\frac{b \epsilon_{\mathrm{o}}}{2}\left[\frac{\epsilon V}{\epsilon_{\mathrm{o}} d_{\mathrm{e}}+\epsilon(g-w)}\right]^{2},
$$

where $\epsilon_{\mathrm{o}}$ is the vacuum permittivity, $\epsilon$ is the permittivity of coated dielectric layer material, $d_{\mathrm{e}}$ is dielectric material layer thickness, $V$ is the voltage between the substrate and the microbeam, and $g$ is the gap distance between the microbeam and dielectric layer.

The energy functional is

$$
\Pi(t, x, S)=T-U_{\mathrm{B}}-U_{S 1}-U_{S 2}+W_{\mathrm{S}}+W_{\mathrm{E}} .
$$

By applying Hamilton's principle $\left(\int_{t_{1}}^{t_{2}} \delta \Pi \mathrm{d} t=0\right)$, the governing equation for the microbeam in $0 \leqslant x \leqslant S$ domain is derived as

$$
\rho w_{t t}+E I \frac{\partial^{4} w}{\partial x^{4}}-\left[\frac{A E}{2 S} \int_{0}^{S}\left(\frac{\partial w}{\partial x}\right)^{2} \mathrm{~d} x-P\right] \frac{\partial^{2} w}{\partial x^{2}}=f_{\mathrm{e}} .
$$

And the four boundary conditions are

$$
\left.w\right|_{x=0}=0,\left.\quad \frac{\partial w}{\partial x}\right|_{x=0}=0,\left.\quad w\right|_{x=S}=g,\left.\quad \frac{\partial w}{\partial x}\right|_{x=S}=0 .
$$

The governing equation (10) can only be used to compute the microbeam deflection $w$ in the domain of $0 \leqslant x \leqslant S$. It does not determine $S$. For this dynamic analysis, 
$\partial \Pi / \partial S=0$ is still used to determine $S$ as Mastrangelo and Hsu did for the static case $[10,11]$. For the static case of microbeam structure (with no electrical force influence), there is only one stationary point of $\partial \Pi / \partial S[10,11]$. Zhang and Zhao [9] show that in the static case of the microbeam under an electrostatic force, there is also only one stationary point. For numerical computation, we adjust equation $\partial \Pi / \partial S=0$ to the following equation to determine $S$

$$
\left|\frac{\partial \Pi}{\partial S}\right|<\Delta
$$

where $\Delta$ is a very small positive number. If equation (12) is not satisfied, $S$ is varied to compute $w$, and $\Pi$ again using equations (10) and (9), respectively, because $w$ and $\Pi$ are different for different $S$ and varying the $S$ is the way to satisfy equation (12). The iteration continues until equation (12) is satisfied. Once equation (12) is satisfied, $S$ is found and $w$ is re-computed one more time. To illustrate this procedure of finding $S$, a simple static example with no electrical force, no axial load and no nonlinearity due to axial stretching, is given. For this case, the governing equation (10) changes as

$$
E I \frac{\partial^{4} w}{\partial x^{4}}=0, \quad 0 \leqslant x \leqslant S .
$$

The boundary conditions remain the same as those in equation (11). The microbeam deflection $w(x, S)$ is determined as

$$
w=-\frac{2 g}{S^{3}} x^{3}+\frac{3 g}{S^{2}} x^{2}
$$

Here $S$ is an unknown constant. Now because $T=U_{S 1}=U_{S 2}=W_{\mathrm{E}}=0$, $\Pi=-U_{\mathrm{B}}+W_{\mathrm{S}}=-\left(\left(6 E I g^{2}\right) / S^{3}\right)+b(L-S) f_{\theta} W_{\mathrm{a}}$. To determine $S$, we take the derivative

$$
\frac{\partial \Pi}{\partial S}=\frac{18 E I g^{2}}{S^{4}}-b f_{\theta} W_{\mathrm{a}}=0 .
$$

From equation (15), $S$ is determined as

$$
S=\left(\frac{18 E I g^{2}}{b f_{\theta} W_{\mathrm{a}}}\right)^{1 / 4}=\left(\frac{3 E h^{3} g^{2}}{2 f_{\theta} W_{\mathrm{a}}}\right)^{1 / 4} .
$$

Because of the simplicity of this problem, here $S$ is directly solved without iteration. This $S$ value is the long slender microbeam equilibrium unstuck length with the peel number $N_{\mathrm{p}}=1[10,11,17]$. The peel number is the ratio of the elastic energy of the deformed microstructure to the the work of adhesion between the microstructure and substrate $[10,11]$. For $N_{\mathrm{p}}>1$, the beam elastic energy is greater than the work of adhesion, and thus the beam cannot adhere to the substrate. For $N_{\mathrm{p}} \leqslant 1$, the beam adheres to the substrate [17]. 


\section{NON-DIMENSIONALIZATION AND NUMERICAL METHOD}

To non-dimensionalize the equations, the following quantities are introduced [6]

$$
W=\frac{w}{g}, \quad \xi=\frac{x}{L}, \quad R=\frac{S}{L}, \quad \tau=\sqrt{\frac{E I}{\rho L^{4}}} t .
$$

The governing equation (10) now becomes

$$
W_{\tau \tau}+W^{\prime \prime \prime \prime}-\left[\frac{\alpha_{1}}{R} \int_{0}^{R}\left(W^{\prime}\right)^{2} \mathrm{~d} \xi-\alpha_{2}\right] W^{\prime \prime}=\frac{\alpha_{3} V^{2}}{(W-\beta)^{2}} .
$$

Here

$$
\alpha_{1}=6\left(\frac{g}{t}\right)^{2}, \quad \alpha_{2}=\frac{P g}{E I L^{2}}, \quad \alpha_{3}=\frac{6 \epsilon_{\mathrm{o}} L^{4}}{E g^{3} t^{3}} \quad \text { and } \beta=1+\frac{d_{\mathrm{e}}}{g \epsilon_{\mathrm{r}}} .
$$

$\epsilon_{\mathrm{r}}$ is relative permittivity defined as $\epsilon_{\mathrm{r}}=\epsilon / \epsilon_{\mathrm{o}}$. Here the operator ()$^{\prime}=\partial / \partial \xi$. Now, the boundary conditions are

$$
\left.W\right|_{\xi=0}=0,\left.\quad \frac{\partial W}{\partial \xi}\right|_{\xi=0}=0,\left.\quad W\right|_{\xi=R}=1,\left.\quad \frac{\partial W}{\partial \xi}\right|_{\xi=R}=0 .
$$

The functional $\Pi$ now becomes

$$
\begin{gathered}
\Pi=\Pi(\tau, \xi, R)=\frac{E I g^{2}}{2 L^{3}}\left\{\int_{0}^{R} W_{\tau}^{2} \mathrm{~d} \xi-\int_{0}^{R}\left(W^{\prime \prime}\right)^{2} \mathrm{~d} \xi-\frac{\alpha_{1}}{2 R}\left[\int_{0}^{R}\left(W^{\prime}\right)^{2} \mathrm{~d} \xi\right]^{2}\right\} \\
-\frac{E I g^{2}}{2 L^{3}}\left[\alpha_{2} \int_{0}^{R} W^{\prime 2} \mathrm{~d} \xi\right. \\
\left.+2 \alpha_{3} V^{2} \int_{0}^{R}\left(\frac{-1}{\beta}-\frac{1}{W-\beta}\right) \mathrm{d} \xi+\alpha_{4}(1-R)\right]
\end{gathered}
$$

Here

$$
\alpha_{4}=\frac{2 b L^{4} f_{\theta} W_{\mathrm{a}}}{E I g^{2}}=\frac{24 L^{4} f_{\theta} W_{\mathrm{a}}}{E h^{3} g^{2}} .
$$

For $\partial \Pi / \partial S=(1 / L) \partial \Pi / \partial R$, the expression is extremely lengthy and, thus, is not given here. The general rule of how to take derivative $(\partial / \partial R)$ of functional $\int_{a(R)}^{b(R)} f(\xi, R) \mathrm{d} \xi$ is given [9].

$W$ is assumed to have the following expression

$$
W(\tau, \xi, R)=W_{\mathrm{o}}(\xi, R)+U(\tau, \xi, R)=W_{\mathrm{o}}(\xi, R)+\sum_{i=1}^{N} a_{i}(\tau) \phi_{i}(\xi, R) .
$$

Here $W_{\mathrm{o}}(\xi, R)$ is the solution of

$$
W_{\mathrm{o}}^{\prime \prime \prime \prime}=0,
$$


with the boundary conditions of

$$
\left.W_{\mathrm{o}}\right|_{\xi=0}=0,\left.\quad \frac{\partial W_{\mathrm{o}}}{\partial \xi}\right|_{\xi=0}=0,\left.\quad W_{\mathrm{o}}\right|_{\xi=R}=1,\left.\quad \frac{\partial W_{\mathrm{o}}}{\partial \xi}\right|_{\xi=R}=0 .
$$

From equations (21) and (22), it is not that difficult to conclude that $W_{\mathrm{o}}$ is the equilibrium of the linear $\mathrm{S}$-shaped microbeam with no axial load and no electrical force. $a_{i}(\tau)$ is a time-dependent variable (it is the constant to be determined in each time step). $N$ is the mode number. $\phi_{i}(\xi, R)$ is the $i$ th mode shape solved from the following governing equation:

$$
U_{\tau \tau}+U^{\prime \prime \prime \prime}=0
$$

and with the boundary conditions of the fixed-fixed beam

$$
\left.U\right|_{\xi=0}=0,\left.\quad \frac{\partial U}{\partial \xi}\right|_{\xi=0}=0,\left.\quad U\right|_{\xi=R}=0,\left.\quad \frac{\partial U}{\partial \xi}\right|_{\xi=R}=0 .
$$

By using the separation of variables method and applying boundary conditions, $\phi_{i}(\xi, R)$ is given by Chang and Craig [19] as

$$
\phi_{i}(\xi, R)=\frac{1}{2}\left(\delta_{i} e^{\frac{c_{i} \xi}{R}}+\gamma_{i} e^{-\frac{c_{i} \xi}{R}}\right)-\cos \left(\frac{c_{i} \xi}{R}\right)+\kappa_{i} \sin \left(\frac{c_{i} \xi}{R}\right),
$$

$c_{i}=f_{i} R$ and $f_{i}^{4}=\Omega_{i}^{2}\left(\Omega_{i}\right.$ is the $i$ th dimensionless resonant circular frequency). $c_{i}, \delta_{i}, \gamma_{i}$ and $\kappa_{i}$ are the given constants. For a higher beam mode, the mode shape $\phi_{i}$ above can be simplified. The asymptotic approximation and simple expression for the mode shapes are given by Dowell [20] and Dugundji [21]. It is clear that from the boundary conditions of $W_{\mathrm{o}}$ and $U$, the boundary conditions of $W$ (equation (18)) are satisfied. 
By substituting $W=W_{\mathrm{o}}+\sum_{i=1}^{N} a_{i}(\tau) \phi_{i}(\xi, R)$, multiplying $\phi_{i}$ and integrating from 0 to $R$ in equation (17), the following set of $N$ equations is obtained

$$
\begin{aligned}
& \iint_{0}^{R} \phi_{1} \sum_{i=1}^{N} \ddot{a}_{i} \mathrm{~d} \xi+\int_{0}^{R} \phi_{1}\left\{\sum_{i=1}^{N} a_{i} \phi_{i}^{\prime \prime \prime \prime} \mathrm{d} \xi-\left[\frac{\alpha_{1}}{R} \int_{0}^{R}\left(\sum_{i=1}^{N} a_{i} \phi_{i}^{\prime}\right)^{2} \mathrm{~d} \xi-\alpha_{2}\right]\right. \\
& \left.\times\left(W_{\mathrm{o}}^{\prime \prime}+\sum_{i=1}^{N} a_{i} \phi_{i}^{\prime \prime}\right)\right\} \mathrm{d} \xi \\
& =\int_{0}^{R} \phi_{1} \frac{\alpha_{3} V^{2}}{\left(W_{\mathrm{o}}+\sum_{i=1}^{N} a_{i} \phi_{i}-\beta\right)^{2}} \mathrm{~d} \xi \\
& \int_{0}^{R} \phi_{2} \sum_{i=1}^{N} \ddot{a}_{i} \mathrm{~d} \xi+\int_{0}^{R} \phi_{2}\left\{\sum_{i=1}^{N} a_{i} \phi_{i}^{\prime \prime \prime \prime} \mathrm{d} \xi-\left[\frac{\alpha_{1}}{R} \int_{0}^{R}\left(\sum_{i=1}^{N} a_{i} \phi_{i}^{\prime}\right)^{2} \mathrm{~d} \xi-\alpha_{2}\right]\right. \\
& \left.\times\left(W_{\mathrm{o}}^{\prime \prime}+\sum_{i=1}^{N} a_{i} \phi_{i}^{\prime \prime}\right)\right\} \mathrm{d} \xi \\
& =\int_{0}^{R} \phi_{2} \frac{\alpha_{3} V^{2}}{\left(W_{\mathrm{o}}+\sum_{i=1}^{N} a_{i} \phi_{i}-\beta\right)^{2}} \mathrm{~d} \xi \\
& \int_{0}^{R} \phi_{N} \sum_{i=1}^{N} \ddot{a}_{i} \mathrm{~d} \xi+\int_{0}^{R} \phi_{N}\left\{\sum_{i=1}^{N} a_{i} \phi_{i}^{\prime \prime \prime \prime} \mathrm{d} \xi-\left[\frac{\alpha_{1}}{R} \int_{0}^{R}\left(\sum_{i=1}^{N} a_{i} \phi_{i}^{\prime}\right)^{2} \mathrm{~d} \xi-\alpha_{2}\right]\right. \\
& \left.\times\left(W_{\mathrm{o}}^{\prime \prime}+\sum_{i=1}^{N} a_{i} \phi_{i}^{\prime \prime}\right)\right\} \mathrm{d} \xi \\
& =\int_{0}^{R} \phi_{N} \frac{\alpha_{3} V^{2}}{\left(W_{\mathrm{o}}+\sum_{i=1}^{N} a_{i} \phi_{i}-\beta\right)^{2}} \mathrm{~d} \xi .
\end{aligned}
$$

Here $\dot{(})=\partial() / \partial \tau$ and the fact that $\partial\left[W_{\mathrm{o}} /(\xi, R)\right] / \partial \tau=0$ are used.

\section{RESULTS AND DISCUSSION}

$F_{\mathrm{e}}$, the nondimensionalized shaking electrical force per unit length, can be written as

$$
F_{\mathrm{e}}=\frac{\alpha_{3} V^{2}}{(W-\beta)^{2}}=\frac{\alpha_{3} V_{\mathrm{o}}^{2} \sin ^{2}(\Omega \tau)}{(W-\beta)^{2}}=\frac{\alpha_{3} V_{\mathrm{o}}^{2}[1-\cos (2 \Omega \tau)]}{2(W-\beta)^{2}} .
$$

We call $\alpha_{3} V_{\mathrm{o}}^{2}$ the shaking amplitude and $2 \Omega$ the shaking frequency for convenience. The parameter $\beta$ is taken as 1.1 for all computation cases. $F_{\mathrm{e}}$ is dependent on the 
displacement $W$ and its effect on the adhered microbeam flexural motion is also implicitly dependent on $R$. During the vibration, $R$ changes constantly as time changes. Therefore, the governing equation domain changes. As a result, the system's natural frequencies also change with time. Thus, the microbeam cannot reach a steady state and the resonance is not expected, even when the shaking lasts long enough.

We define the dimensionless length of the unadhered microbeam part, $R_{\mathrm{o}}$, as follows

$$
R_{\mathrm{o}}=\frac{S_{\mathrm{o}}}{L}=\frac{\left(\frac{3 E h^{3} g^{2}}{2 f_{\theta} W_{\mathrm{a}}}\right)^{1 / 4}}{L}=\left(\frac{36}{\alpha_{4}}\right)^{1 / 4},
$$

$S_{0}$, the length of unadhered beam part, is given in the illustration example. It is noticed that in that example, it is linear and there is no axial or electrical load. Because $F_{\mathrm{e}}=0$ at $\tau=0$ and if the initial modal amplitude $a_{i}$ values and initial modal velocity $\dot{a}_{i}$ values are all set as zero, the analytical solution $R_{\mathrm{o}}$ is the solution for $R$ at $\tau=0$ when $\alpha_{1}=\alpha_{2}=0$. By setting both $\alpha_{1}$ and $\alpha_{2}$ zero, $R_{\mathrm{o}}$ can serve as the verification value to check whether the model and computation work properly at the beginning. This paper aims to study the basic behavior of dynamic adhesion and also for this verification reason, $\alpha_{1}$ and $\alpha_{2}$ are both set to be zero. And all modal amplitudes and velocities are also set to be zero as the initial conditions. The Hilbert-Hughes-Taylor (HHT) alpha method [22-24], which offers good numerical stability control, is used for the time integration.

In Fig. 2, $\alpha_{4}$ is taken as 576. From the $R_{\mathrm{O}}$ expression given above, $R_{\mathrm{O}}$ is calculated as 0.5 . When the initial conditions $a_{i}$ values and $\dot{a}_{i}$ values are taken as 0 , the initial value of $R$ is supposed to match the static value of $R_{\mathrm{o}}$. Therefore, this 0.5 value serves as the verification number to tell whether the model and program work correctly. Figure 2 shows the $R$ convergence of different modes computational results. The shaking amplitude is $\alpha_{3} V_{\mathrm{o}}^{2}=30$ and the shaking frequency $\left(2 \Omega=2 \Omega_{1}=178.9832\right)$ is between the first natural frequency $\Omega_{1}$ (89.4916) and the second natural frequency $\Omega_{2}$ (246.67484) of the microbeam with $R=R_{\mathrm{o}}=0.5$. $\Omega_{i}$ is the $i$ th natural frequency of the microbeam solved from linear equations (23) and (24) with no electrical force and $R=R_{0}$. Here, $\Omega_{i}$ can serve as a reference frequency for the purpose of the comparison study. AbdelRahman et al. [6] demonstrate that, for the fixed-fixed beam, the system natural frequencies reduce as electrostatic force increases. As the shaking electrical force $F_{\mathrm{e}}$ is a nonlinear function of $W$, the system resonant frequencies are expected to have some shifts from $\Omega_{i}$ values. For this so-called non-ideal energy source system, whose external driving force is a function of the state $(W, \dot{W}, \ddot{W})$, the reader should refer to Nayfeh and Mook's book [25] for further in-depth reading. For the small vibration studied here, $\Omega_{i}$ values can still serve well as reference frequencies for the system response study when the external driving force does not dramatically influence the system natural frequencies. Figure 2 shows the comparison of 5- and 


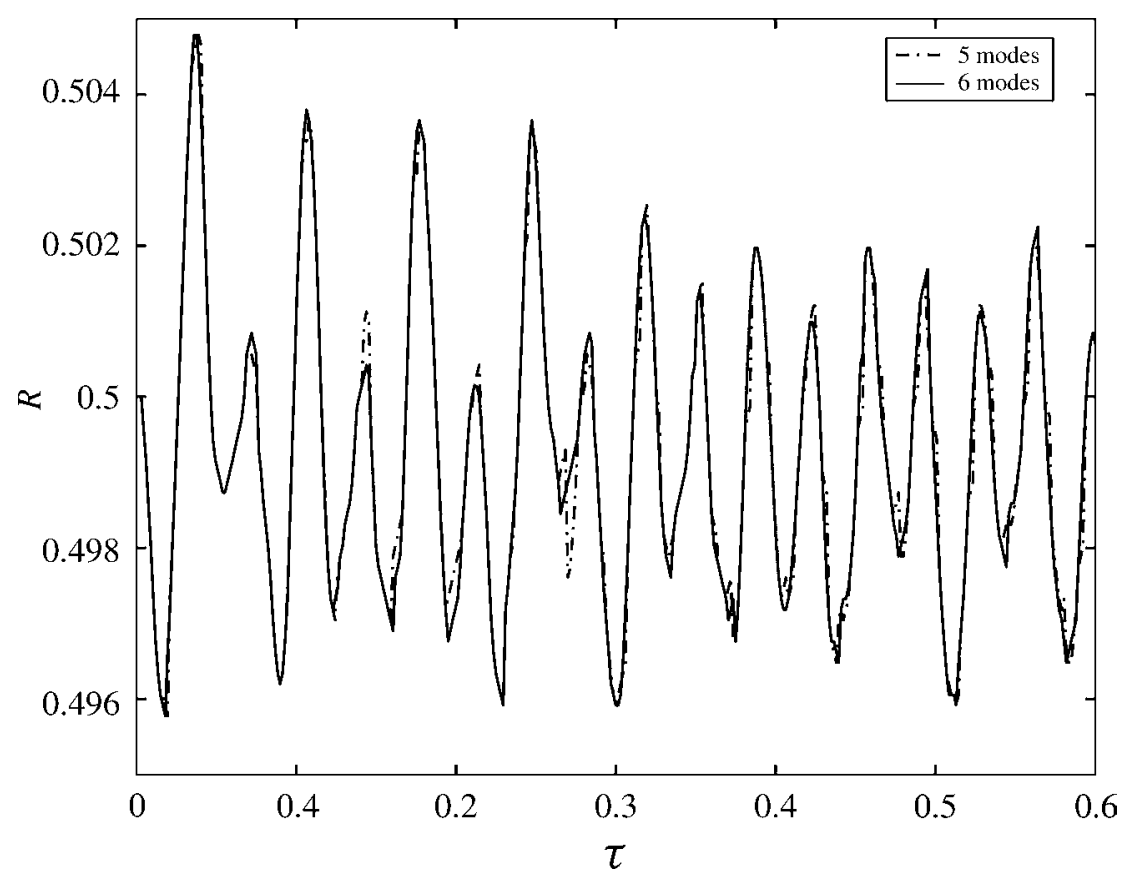

Figure 2. Comparison of $R$ computed under two different mode numbers (5 and 6) when $\Omega=\Omega_{1}$ and $\alpha_{3} V_{\mathrm{o}}^{2}=30$.

6-mode results, and $R$ converges relatively well. Clearly $R$ continuously changes with time. Figure 3 shows the convergence study of the displacement at $\xi=0.3$. Because the shaking frequency $\left(2 \Omega_{1}\right)$ is selected between the first and the second resonant frequencies, the first and second modes are the two major participating modes in the microbeam deflection and our computation confirms that. However, as shown in Figs 2 and 3, the other modes have some influence on the convergence, especially on $R$. The computational error of the complex forms of $\Pi$, its derivative and choice of $\Delta$ value are the major error sources for the computation of $R$. In each time step, the program is designed to let $R$ move back and forth with a certain step size to find a new $R$ around the previous $R$, which can satisfy $|\partial \Pi / \partial R|<\Delta$. If $\Delta$ is very small, a very small step size and a lot of iterations are required to achieve $|\partial \Pi / \partial R|<\Delta$. If $\Delta$ is large, the accuracy of $R$ will be doubtful. Except for some small parts of $R(\tau)$ in Fig. 2, the computation difference between 5 and 6 modes is very small. In Fig. 3, the computation difference between 5 and 6 modes is much smaller. For all the following computation results, the mode number chosen is 6 .

Figure 4 shows the whole beam deflection at time $\tau=0\left(R=R_{\mathrm{O}}=0.5\right)$, $\tau=0.039(R=0.504507)$ and $\tau=0.159(R=0.49704223)$. At $\tau=0$, the initial deflection is $W_{\mathrm{o}}$, which is the analytical available solution of the static case. If looked carefully, the beam deflection curve for $\tau=0.159$ crosses the beam deflection curve for $\tau=0$. This is due to the fact that at $\tau=0.159$, the second modal amplitude is relatively larger. Figure 5 shows the comparison of $R$ under 


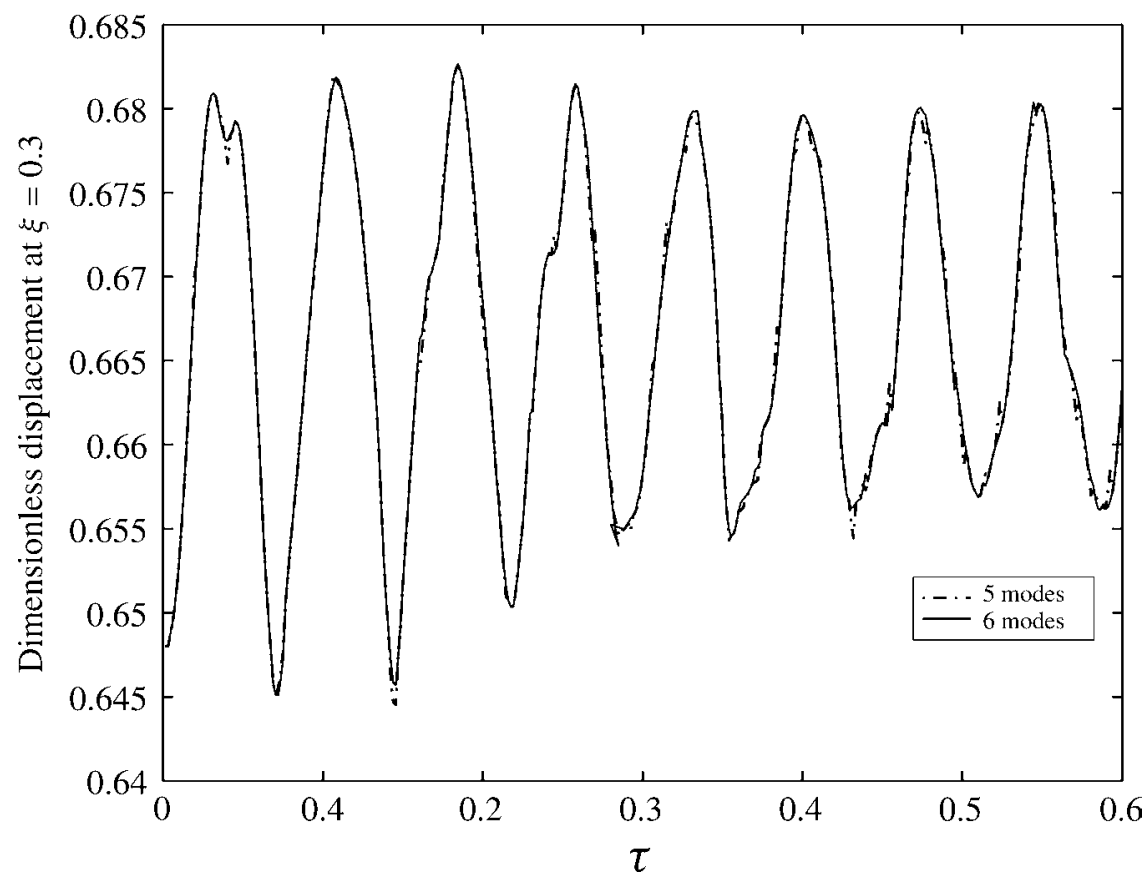

Figure 3. Comparison of displacements at $\xi=0.3$ computed under two different mode numbers (5 and 6) when $\Omega=\Omega_{1}$ and $\alpha_{3} V_{\mathrm{o}}^{2}=30$.

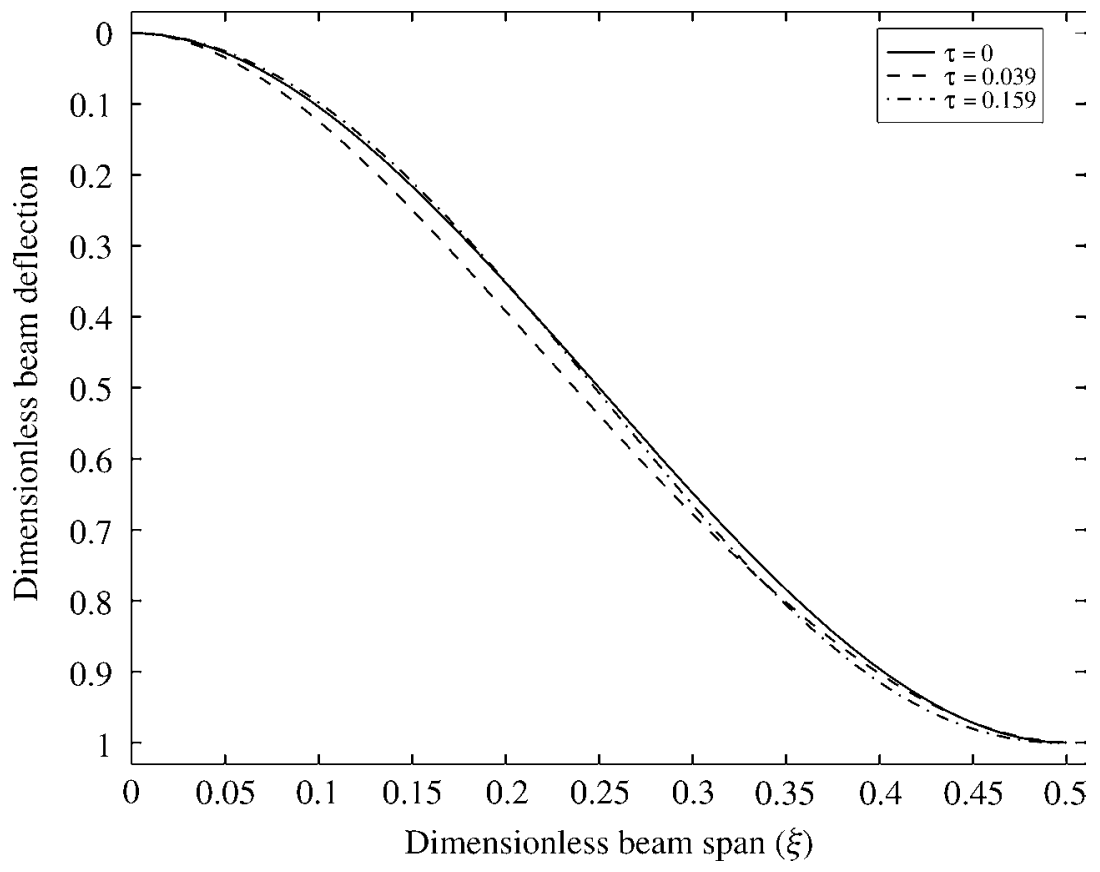

Figure 4. Beam deflection as a function of time when $\Omega=\Omega_{1}$ and $\alpha_{3} V_{\mathrm{o}}^{2}=30$. 


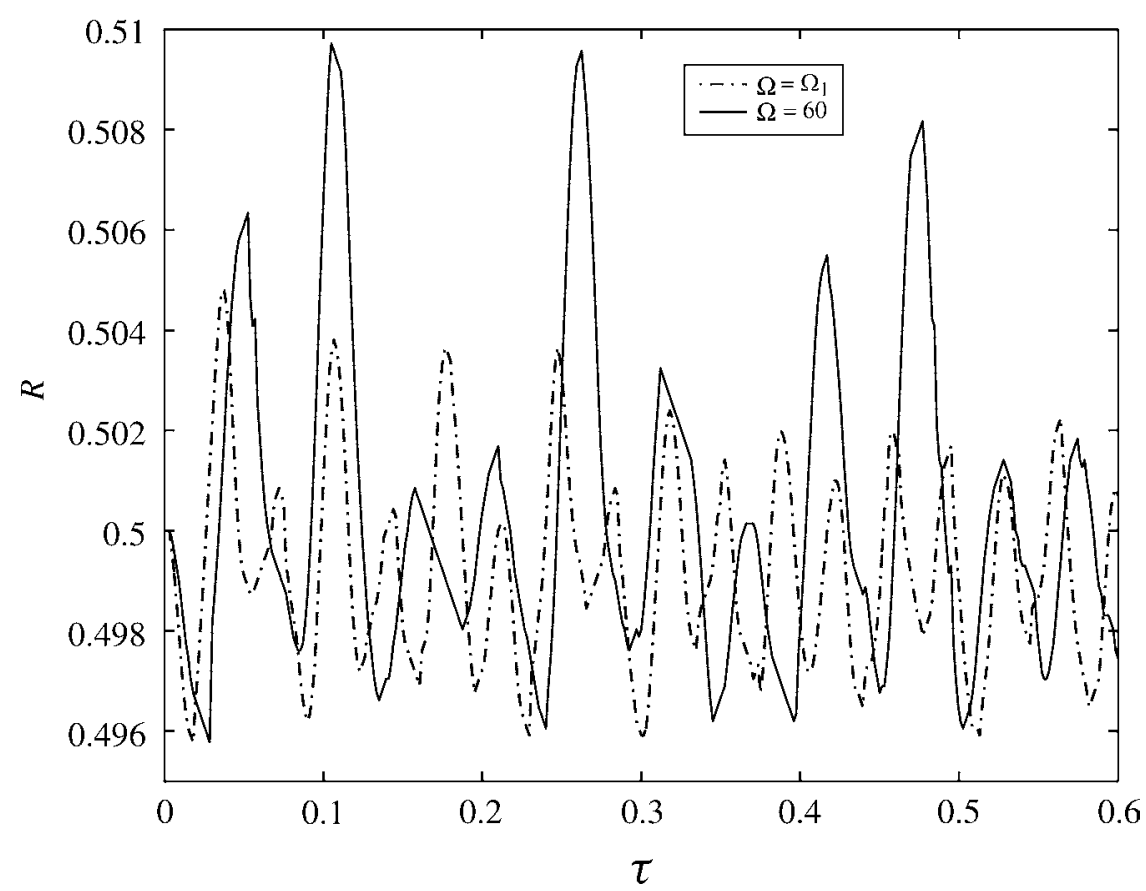

Figure 5. Comparison of $R$ under two different shaking frequencies $\Omega=\Omega_{1}$ and $\Omega=60$ with the same shaking amplitude $\alpha_{3} V_{\mathrm{o}}^{2}=30$.

the two different shaking frequencies $2 \Omega=2 \Omega_{1}$ and $2 \Omega=2 \times 60$ cases. The shaking amplitude $\left(\alpha_{3} V_{\mathrm{o}}^{2}\right)$ for both cases is taken as 30. For the shaking frequency of $2 \Omega=2 \Omega_{1}, 0.5-0.0045 \leqslant R \leqslant 0.5+0.052$. For the shaking frequency of $2 \Omega=2 \times 60,0.5-0.0042 \leqslant R \leqslant 0.5+0.098$. It is asymmetric, which physically means that under the periodically shaking force, the microbeam tends more to peel-off than to adhere. Figure 6 shows the comparison of displacement at $\xi=0.3$ of $2 \Omega=2 \Omega_{1}$ and $2 \Omega=2 \times 60$ cases. $2 \Omega=2 \times 60$ case is closer to the natural frequency $\Omega_{1}=89.4916$ and, thus, a larger response amplitude is shown. It is worth pointing out that, although there is a significant difference in their shaking frequencies and their $R$ responses, the peaks and valleys of their displacement response match each other relatively well as if they share the same response frequency all the time. For a linear system, the steady-state response is directly associated with the shaking force amplitude and frequency. In Fig. 6, the response displacement amplitude difference is very significant but there is only a little frequency response difference for the microbeam under two different shaking frequencies. The fact that $R$ changes continuously, which continuously changes the domain of governing equation (26) and, thus, the natural frequencies of the system. Prevents the system to reach a steady state because a steady state can only be reached for the system with the fixed domain and the natural frequencies when the transients die out. But it still cannot explain why the two microbeam 


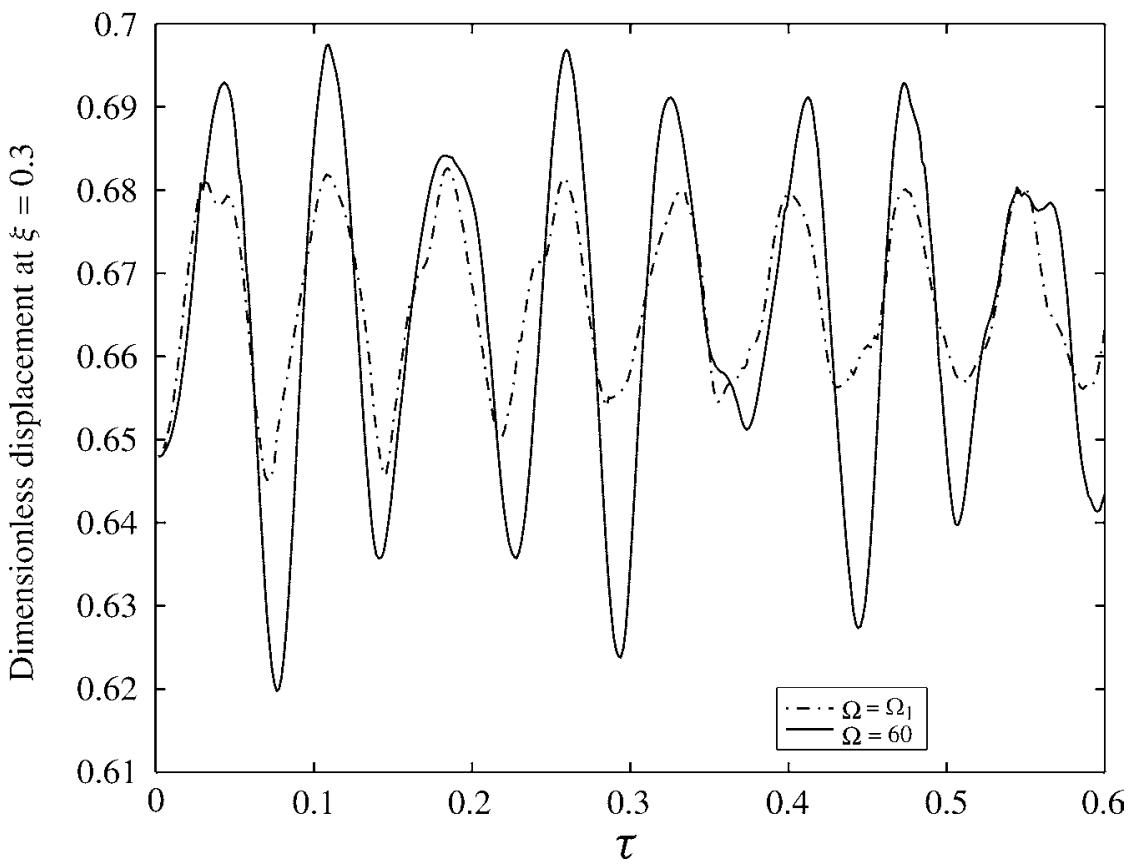

Figure 6. Comparison of displacements at $\xi=0.3$ under two different shaking frequencies $\Omega=\Omega_{1}$ and $\Omega=60$ with the same shaking amplitude $\alpha_{3} V_{\mathrm{o}}^{2}=30$.

response frequencies under the two different shaking frequencies are almost the same. Figure 7 shows the whole beam deflection when $2 \Omega=2 \times 60$ at different times $\tau=0\left(R=R_{\mathrm{O}}=0.5\right), \tau=0.1095(R=0.509296)$ and $\tau=0.2925$ $(R=0.4976056)$.

In Figs $2-7$, it is clearly shown that the adhered microbeam vibrates with very small amplitudes. The shaking frequencies $2 \Omega\left(2 \Omega=2 \Omega_{1}, 2 \times 60\right)$ are deliberately chosen to be away from the resonant frequencies $\Omega_{1}$ and $\Omega_{2}$ (although $R$ changes during the vibration, its effect on natural frequencies change is relatively small). Therefore, only a small vibration response amplitude is expected. Mathematically, nothing keeps us from choosing the shaking frequency closer or equal to the natural/resonant frequencies, but physically it may be problematic. It is noticed that in the governing equation for the unadhered microbeam part, there is no damping term. It is not that difficult to incorporate a $c_{\mathrm{d}} w_{\mathrm{t}}$ ( $c_{\mathrm{d}}$ is the damping coefficient) term in equation (10) to account for the damping effect due to the beam internal friction and the viscosity of the surrounding air. This will not cause any significant change in Figs 2-7. Under forced vibration, the damping coefficient influences the system response amplitude and phase shift and such influence is continuous as damping coefficient changes. During the vibration, especially when the vibrating amplitude is large, the impact and the peeling-off/adhering interaction between the microbeam and substrate will account for the most energy dissipation. And so far, we do not have the dissipation model to account for such (local) energy dissipation 


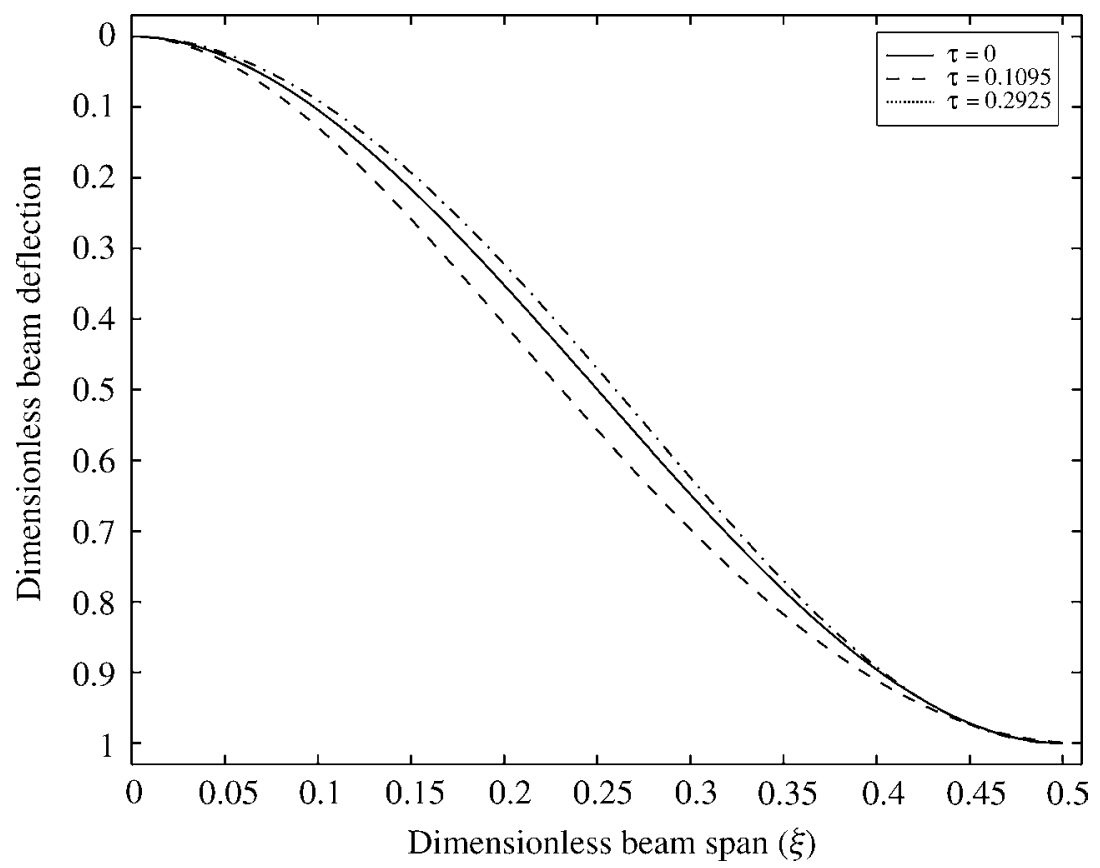

Figure 7. Beam deflection as a function of time when $\Omega=60$ and $\alpha_{3} V_{\mathrm{o}}^{2}=30$.

mechanism. Therefore, for our dynamic computation, our shaking amplitude is chosen to be relatively small and shaking frequency is away from the resonant frequencies to avoid large amplitude response scenario and the modeling difficulties. Also, for such large amplitude vibration case, the vibro-impact model [26-28] should be considered. In the vibro-impact model, the coefficient of restitution is responsible for the energy loss due to the impact [26, 27]. While, applying the method of coefficient of restitution should be restricted to the case of impact object(s) with very large stiffness and under such method, the object is assumed to bounce off immediately [26, 27].

When $\partial \Pi / \partial S=0$ is used to determine the adhesion length, it determines the adhesion length in the $N_{\mathrm{p}}=1$ case only. In reality, the structure adheres when $N_{\mathrm{p}} \leqslant 1$. So our dynamic computation is more like a limit case computation. Van Spengen et al. [29] propose the method to model the real adhesion area as a distribution function by considering the surface roughness effect to solve such peel number issues. The work of adhesion term, equation (5) shows that $b(L-S)$ is taken as the adhesion area and a constant work of adhesion per unit area is assumed in this paper. The difficulty in treating the adhesion area as a distribution function is how to guarantee the computation continuity in each time step because our governing equations are deterministic and the adhesion area keeps changing not only for the shaking force but also for the random process. The shaking force is a continuous function of time, but the random process is not. 
From the fracture mechanics viewpoint on this adhesion problem (the crack front is at $\xi=R)[13,14]$, our approach is a quasistatic one. An elastodynamics approach is very difficult. Depending on the loading conditions, the material characteristics and geometric configuration of body, inertial effects can be very significant [30]. Stress intensity factor (toughness) can be a time-dependent dynamic intensity factor (toughness) under certain loading conditions. Furthermore, the deformations of a crack front change the stresses at the distant points from the front [31] and the stresses are also modified by the boundary conditions of the rough crack surface, which results in the dependence on the crack front history [32]. Especially the normal stresses generated at the microbeam and substrate surfaces will directly influence the beam flexural motion. Such normal stresses are zero in the quasistatic assumption of the crack motion [32]. Unlike usual crack propagation in which the plastic deformation prevents rehealing (crack cannott move backward), $R$ moves back and forth (which physically means the crack moves back and forth).

In Fig. 5, it is shown that $R$ moving frequency is proportional to the shaking frequency and its amplitude is proportional to the shaking frequency 'closeness' to the resonant frequency. Quasistatic assumption is viable when the effective mobility of crack front is very small [32] and this is the physical reason why the shaking amplitude and frequency are chosen to be relatively small and away from the resonant frequencies.

During the computation, $W_{\mathrm{a}}$, the work of adhesion per unit area, is assumed to be constant. Such an assumption may not be applicable to the peeling dynamics of some organic monolayers. For those organic monolayers, depending on their nature, the increase of pull-off force is observed when the driving velocity is increased [33] and a velocity-dependent adhesion model is proposed to explain such phenomenon [34]. As explained by Barthel and Roux, the velocity-dependent adhesion is due to the viscous dissipation, i.e., the layer is fluidlike; and for solidlike layer, there is no such phenomenon [34].

\section{CONCLUSIONS}

We studied the vibration of an adhered microbeam. The excitation parameters are carefully chosen under the awareness of the model limitations. The static approach of determining the structure adhesion length under no electrical force by finding the functional stationary point(s) is extended to the dynamic case of the structure under periodically shaking electrical force. Also, the continuity of adhesion length and the displacement of (an arbitrary) point on the unadhered microbeam part is shown. The model presented here is capable of handling a non-linear large amplitude vibration case, but for reasons of verification and physical limitations of the model, only small vibration cases are shown (the electrical shaking force expression makes the problem nonlinear even for small amplitude vibration cases). The unadhered/adhered beam length is determined through finding the stationary point(s) of functional $\Pi$, which continuously changes with time and results in the 
continuous change of unadhered/adhered length. The equation of motion is for the domain of unadhered part of the beam. The constant domain change is responsible for the system not reaching a steady state, no matter how long the time is. Whether there is damping or impact influence, this qualitative fact will not change. The discussion of the model limitations constitutes a major part of this paper, and the related works to improve the model to be applicable to a general case are also suggested during the discussion of the model limitations.

\section{Acknowledgements}

This research was supported by the Distinguished Young Scholar Fund of the National Natural Science Foundation of China (NSFC, Grant No. 10225209), a key project of Chinese Academy of Sciences (Grant No. KJCX-SW-L2) and NSF-RGC joint project (Grant No. 50131160739).

\section{REFERENCES}

1. F. Yang, J. Appl. Phys. 92, 2789 (2002).

2. E. Chan, K. Carikipati and R. W. Dutton, J. Microelectromech. Syst. 8, 208 (1999)

3. S. G. Adams, F. M. Bertsch, K. A. Shaw, P. G. Hartwell, F. C. Moon and N. C. MacDonald, J. Micromech. Microeng. 8, 15 (1997).

4. W. M. van Spengen, R. Puers and I. De Wolf, J. Micromech. Microeng. 12, 702 (2002).

5. P. M. Osterberg and S. D. Senturia, J. Microelectromech. Syst. 6, 107 (1997).

6. E. M. Abdel-Rahman, M. I. Younis and A. H. Nayfeh, J. Micromech. Microeng. 12, 759 (2002).

7. E. S. Hung and S. D. Senturia, J. Microelectromech. Syst. 8, 497 (1999).

8. H. A. Tilmans and R. Legtenberg, Sensors Actuators A 45, 67 (1994).

9. Y. Zhang and Y. P. Zhao, Acta Mech. Sol. Sin. 17, 104 (2004).

10. C. H. Mastrangelo and C. H. Hsu, J. Microelectromech. Syst. 2, 33 (1993).

11. C. H. Mastrangelo and C. H. Hsu, J. Microelectromech. Syst. 2, 44 (1993).

12. N. R. Tas, C. Gui and M. Elwenspoek, J. Adhesion Sci. Technol. 17, 547 (2003).

13. E. E. Jones, M. R. Begle and K. D. Murphy, J. Mech. Phys. Solids 51, 1601 (2003).

14. M. P. de Boer and T. A. Michalske, J. Appl. Phys. 86, 817 (1999).

15. M. P. de Boer, J. A. Knapp, T. M. Mayer and T. A. Michalske, in: Proc. SPIE, Vol. 3825, p. 2 (1999).

16. P. H. McDonald, J. Appl. Mech. 22, 573 (1955).

17. Y. P. Zhao, L. S. Wang and T. X. Yu, J. Adhesion Sci. Technol. 17, 519 (2003).

18. H. H. Yu and Z. Suo, J. Mech. Phys. Solids 46, 829 (1998).

19. T. C. Chang and R. R. Craig, J. Eng. Mech., 195, 1027 (1969).

20. E. H. Dowell, J. Appl. Mech. 51, 439 (1984).

21. J. Dugundji, AIAA J. 26, 1013 (1988).

22. T. J. R. Hughes, The Finite Element Method: Linear Static and Dynamic Finite Element Analysis. Prentice-Hall, Englewood Cliffs, NJ (1987).

23. V. Cannillo and M. Mancuso, J. Sound Vibr. 249, 599 (2002).

24. R. E. Cornwell and D. S. Malkus, Comput. Methods Appl. Mech. Eng. 97, 149 (1992).

25. A. H. Nayfeh and D. T. Mook, Nonlinear Oscillations. Wiley, New York, NY (1979).

26. S. W. Shaw and P. J. Holmes, Phys. Rev. Lett. 51, 623 (1983).

27. S. W. Shaw and P. J. Holmes, J. Sound Vibr. 90, 129 (1983).

28. G. S. Whiston, J. Sound Vibr. 152, 427 (1992). 
29. W. M. van Spengen, R. Puers and I. De Wolf, IEEE Trans. Device Mater. Reliabil. 3, 167 (2003). 30. L. B. Freund, Dynamic Fracture Mechanics. Cambridge University Press, Cambridge (1990). 31. J. R. Rice, J. Appl. Mech. 52, 571 (1985).

32. S. Ramanathan, D. Ertas and D. S. Fisher, Phys. Rev. Lett. 79, 873 (1997).

33. M. Ruths and S. Granick, Langmuir 14, 1804 (1998).

34. E. Barthel and S. Roux, Langmuir 16, 8134 (2000). 